\section{Challenges in Pain Research}

Pain, a health problem that affects a large segment of the population, has a high cost for treatment and management. For some groups of patients, the methods and drugs available are effective, while to others they fall short. Basic research has advanced substantially and provided fundamental theories for understanding the mechanisms of this clinical situation, such as neuroplasticity, gate theory of pain or central and peripheral sensitization. The concepts in these areas were transferred to clinical practice and, despite being based on experimental data, the results were multimodal therapy, pre-emptive analgesia and a better understanding - which resulted in better treatment - of acute pain.

Despite the discovery of several mechanisms involved in the pain process that are susceptible to control with the use of drugs (adenosine receptors, glutamate and aspartate receptors, neurokinin, and others), this knowledge has not resulted in the introduction of novel drugs. The analgesic drugs in use are in the same category of those that already exist, or were brought in from other areas in which drugs such as gabapentin, clonidine, and ketamine were already known.

Clinical pain differs greatly from other conditions, in which the factors involved can be measured objectively. The impact of myocardial infarction can be objectively observed and evaluated. This fact significantly contributed to disease prevention and patient improvement. A number of drugs and invasive techniques for the recovery of hemodynamic, based on research results, provided better evolution of the disease, less discomfort and an increasing survival to the patient. Regarding the subjective factor, an important component of pain observed in humans, it is impossible to be evaluated in experimental animals. Not to mention the neuroanatomic characteristics involved in pain sensation, which are absent in rodents that are models for most pain studies. Even genetically modified animals, increasingly used in experiments, lack the affective aspect of pain and its implications for nociceptive information. Moreover, morbidity with specific characteristics are absent in healthy experimental models. Perhaps the development and/or use of models with diseases common to humans could be one of the paths in the search for new treatments and drugs to control chronic pain. Many non-rodent animals, such as dogs and cats of certain breeds, may have diseases similar to humans. Animals affected by these diseases may be useful models in drug development and understanding of medical conditions. Rodent models have been useful in the study of pain mechanisms, but very inefficient in the development of new drugs for pain treatment in humans. We could ask: are the current rodent models inappropriate for the study of pain or do they lack validity or fidelity to the clinical conditions found in humans? Validity refers to the fact that the chosen model should convincingly measure or reflect what is to be measured. For instance, is there validity in models of peripheral nerve damage induced in rats (sciatic nerve ligation), used as standard in the study of pain, in the development of analgesics for treatment of neuropathic pain? Randomized, controlled clinical trials of analgesics for neuropathic pain were conducted in patients without peripheral neurologic damage. Imaging studies in humans pointed to cortical structures involved in conscious perception of pain. Therefore, one can infer that it is crucial to quantify the behavioral responses mediated by the sensory cortex in laboratory animals. Reflexes, such as withdrawal of tail or limb placed on a hot plate, may not be mediated by cortical structures. The time between the experimental damage induced in animals used for experimentation and the subsequent analysis is much shorter than the human clinical conditions that persist for several years. Even taking into account differences in life span between species, the difference in disease chronicity may have different implications in both scenarios. Given this lack of predictability of animal models of pain, it is important to rethink what can be done to increase tests validity and bring new insights for clinical treatment.

The drug industry needs heavy investments. Costs to develop new drugs are very high. For the industry, it is essential that new products are successful in the market and generate profit. The watchword among manufacturers is to reduce costs and increase efficiency. The process between research and commercialization of a new drug can take up to 20 years. Many of the pharmacological effects described in vitro cannot be confirmed in vivo, and thousands of dollars invested in research have been thrown away. The chances of success in other markets, such as hypertension or erectile dysfunction, are more attractive than the market of pain relief products. As one can imagine, these factors also has its share of impact on the production of new drugs for treatment of painful conditions.

The future of this area is still uncertain. However, a closer relationship between clinical and laboratory researchers, with the consequent exchange of information, is desirable. Studies of gene expression of pain and the development of genetically modified laboratory animals to study this condition are now feasible approaches that could bring results in the future. The number of articles submitted and published by many scientific journals devoted to the subject is large and there are no signs that it should decrease. Therefore, in addition to being compelling, new fields of research and new ways of thinking should be integrated in order to result in advances in the development of new drugs and techniques in clinical pain management.

Mario J Da Conceição, MD, MSc, PhD Professor of Surgical and Anesthetic Techniques Fundação Universidade de Blumenau - FURB

BJAn Editor in chief 